\title{
TEMPERATURA, UMIDADE RELATIVA E DISPONIBILIDADE DE ÁGUA NO ACÚMULO DE PROLINA EM CULTIVARES DE FEIJOEIRO ${ }^{(1)}$
}

\author{
HAIKO ENOK SAWAZAKI $\left({ }^{2,4}\right)$, JOÃO PAULO FEIJĀO TEIXEIRA $\left({ }^{2,4}\right)$ \\ e EDUARDO ANTONIO BULISANI $\left({ }^{3,4}\right.$ )
}

\section{RESUMO}

O estudo đe plantas de feijão (Phaseolus vulgaris L.) submetidas à deficiência hrdrica e temperaturas de $35-40^{\circ} \mathrm{C}$, umidade relativa (UR) $40-100 \% ; 5-10^{\circ} \mathrm{C}$, UR $20-30 \% ; 2-5^{\circ} \mathrm{C}$, UR $10-20 \%$, realizado em casa de vegetação e câmara de crescimento, no Instituto Agronômico, em 1982, mostrou haver correlação negativa entre o acúnulo de prolina e o potencial de pressão de água nas folhas $(\psi p)$. Os cultivares Curitibano e P-511, este último considerado resistente à seca e baixa temperatura, apresentaram o menor acúmulo de prolina e o maior $\psi \mathrm{p}$, confirmando a hipótese de que é possível o reconhecimento de material geneticamente resistente à escassez de água através da seleça de plantas que acumulam menor quantidade de prolina.

Termos de indexação: temperatura, água e umidade; prolina; potencial de água.

\section{INTRODUÇÃo}

A utilização do acúmulo de prolina em p̄lantas como índice de resistência à seca, sustentada por diversos autores, como SINGH et alii (1973); RENA \& MASCIOTTI (1976); JAGER \& MEYER (1977), é baseada no fato de que, sendo a prolina altamente solúvel, pode atuar em concentrações extremas como estoque ativo de carbono, nitrogènio e energia. Entretanto,

\footnotetext{
(1) Recebido para publicação em 5 de junho de 1984.

$\left({ }^{2}\right)$ Seção de Fitoquímica, Instituto Agronômico (IAC), Caixa Postal 28, 13100 Campinas (SP).

$\left({ }^{3}\right)$ Seção de Leguminosas, IAC.

$\left({ }^{4}\right)$ Com bolsa de suplementação do CNPq.
} 
HANSON et alii $(1977,1979$ a, b) contestam essa teoria, afirmando que a alta de acúmulo daquele aminoácido está associada a uma rápida e precoce perda de capacidade de translocar nitrogênio e, possivelmente, carbono, pois não consideraram que a prolina assuma papel importante no transporte de nitrogênio, sendo mais um sintoma de resposta nociva do que adaptação com valor de sobrevivência. Estudos de STEWART et alii $(1977,1978)$; BOGGESS et alii (1978); HUANG \& CAVALIERI (1979), mostraram que (a) a prolina é acumulada em conseqüência de processo catabólico do ciclo de Krebs nas-membranas mitocondriais, a partir de glutamato; (b) ocorre inibição da enzima prolina-oxidase e da síntese de proteína, provocando a elevação da concentração de aminoácidos livres e, (c) ocorre ainda perda da inibição de "feed-back".

Em vista da forte influência do meio ambiente sobre a taxa de desenvolvimento das plantas sob estresse de água e sobre o acúmulo de prolina, as diferenças genéticas somente podem ser detectadas quando genótipos crescem e são submetidos simultaneamente a estresse sob condições idênticas de tratamento e amostragem, segundo HANSON et alii (1979).

Neste trabalho, fez-se um estudo utilizando condições controladas de temperatura e umidade para experimentos que envolveram cultivares de feijão, selecionados, de acordo com dados sobre acúmulo de prolina anteriormente obtidos por SAWAZAKI et alii (1981 a, b), e mais um apontado como resistente à seca pelo Centro Internacional de Agricultura Tropical - CIAT (Cali, Colômbia). Buscou-se verificar a correlação entre potencial de pressão de água nas folhas $\left(\psi_{\mathrm{p}}\right)$ e o nível de prolina acumulada nesse órgão, visando, como objetivo maior, verificar a viabilidade de utilização desse índice como parâmetro químico para seleção genética de plantas mais tolerantes à escassez de água no solo.

\section{MATERIAL E MÉTODOS}

Para estudo do acúmulo de prolina relacionado ao estresse hídrico, foram testados no Instituto Agronômico, em 1982, os seguintes cultivares de feijoeiro: Jalo, Roseli, Bico-de-Ouro, Rosinha-G ${ }_{2}$, Curitibano, Aroana, Moruna e P-511, este último citado como resistente à seca pelo CIAT. Foram realizados quatro experimentos, utilizando plantas desenvolvidas tanto em casa de vegetação quanto em câmara de crescimento com controle de umidade e temperatura, monitorizados através de termoigrógrafo, e vasos com 2 litros de capacidade com quatro plantas cada um e quatro repetições por cultivar. Em todos os experimentos, a amostra para determinação de prolina consistiu em cinco discos de $1 \mathrm{~cm}$ de diâmetro, retirados do limbo foliar de folhas recém-expandidas e imediatamente imersos em solução de metanolclorofórmio-água (MCW) na razão de 12:5:1 (v/v/v), e conservados a $-18^{\circ} \mathrm{C}$. 
Os teores de prolina foram determinados segundo RENA \& MASCIOTTI (1976), em micromoles por grama de matéria seca de folha.

\section{Experimento I}

Os vasos com composto terriço adubado com fósforo e potássio foram colocados em câmara de crescimento regulada para temperatura no intervalo de 20 a $25^{\circ} \mathrm{C}$ e umidade relativa entre 40 e $100 \%$, e 14 horas diárias de luz. Quando as plantas atingiram vinte dias de idade, iniciou-se estresse hídrico por dois e oito dias, período em que as condições de câmara foram reguladas para temperatura de 35 a $40^{\circ} \mathrm{C}$ e umidade relativa de 30 a $100 \%$. Após o estresse de oito dias, os vasos foram irrigados e a recuperação das plantas estimada por mediçóes da altura, avaliada nas idades de 26,33 e 39 dias. Nas plantas amostradas, efetuou-se, além da determinação do acúmulo de prolina, o potencial de água $\left(\psi_{\mathrm{p}}\right)$ na folha através da câmara de Scholander.

\section{Experimento II}

No segundo experimento, procurou-se associar estresse hídrico e baixas temperaturas. Vasos com solo adubado e plantas de 25 dias de idade que se tinham desenvolvido em câmara à temperatura de 20 a $25^{\circ} \mathrm{C}$ e umidade relativa de 40 a $100 \%$ foram transferidos para 5 a $10^{\circ} \mathrm{C}$ e umidade relativa de 20 a $30 \%$ sob iluminação contínua. Nessas condições, aplicou-se estresse hídrico, sendo as plantas amostradas para avaliação do aumento de prolina, dois e onze dias após a última irrigação.

\section{Experimento III}

Foram utilizadas plantas desenvolvidas em vaso com vermiculita e irrigadas com solução completa de Hoagland 1. Os vasos foram transferidos da casa de vegetação para câmara de crescimento regulada a temperaturas de 2 a $5^{\circ} \mathrm{C}$ e umidade relativa de 15 a $20 \%$, quando as plantas tinham 23 e 67 dias de idade. As amostragens para determinação do teor de prolina foram efetuadas após estresse hídrico de onze dias para as plantas de menos idade e treze dias para as mais velhas (florescimento). Nestas foi também medida a altura total.

\section{Experimento IV}

Os vasos com vermiculita e irrigados com solução nutritiva completa de Hoagland 1 foram deixados em casa de vegetação. Quando as plantas estavam com 22 dias de idade, iniciou-se estresse hídrico, efetuando-se as amostragens para acúmulo de prolina 14 dias após. 


\section{RESULTADOS E DISCUSSÃO}

A análise estatística revelou diferenças significativas tanto para o teor de prolina quanto para o potencial de água da folha entre os cultivares após os dois períodos de estresse hídrico (Quadro 1). Aparentemente, o aumento do acúmulo de prolina pode estar relacionado com o maior desenvolvimento das plantas, caso específico dos cultivares Jalo e Carioca (Quadro 2), que, aos 39 dias de idade, apresentavam um elongamento mais acentuado. $\mathrm{O}$ fato da pequena diferença de acúmulo de prolina e da não-correlação com as medidas de $\psi_{\mathrm{p}}$ observadas indica que, apesar da temperatura elevada e suspensão de água, a alta umidade relativa interferiu na escassez de água, que não foi suficiente para diferenciar claramente os cultivares.

QUADRO 1 - Acúmulo de prolina livre ( $\mu \mathrm{mol} / \mathrm{g}$ de matéria seca) e potencial de pressão de água $(\psi \mathrm{p})$ em folhas de feijoeiro com 20 dias de idade não irrigados por 2 e 8 dias

\begin{tabular}{|c|c|c|c|c|c|c|c|c|}
\hline \multirow{3}{*}{ Cultivar } & \multicolumn{8}{|c|}{ Dias sem irrigação } \\
\hline & \multicolumn{4}{|c|}{2} & \multicolumn{4}{|c|}{8} \\
\hline & Prolina & $\mathrm{s}$ & $\psi p$ & $\mathrm{~s}$ & Prolina & $s$ & $\psi \mathrm{p}$ & s \\
\hline & $\mu \mathrm{mol} / \mathrm{g}$ & & atm & & $\mu \mathrm{mol} / \mathrm{g}$ & & atm & \\
\hline Jalo & $2,1 \mathrm{a}$ & 0,6 & $-4,8 c$ & 0,5 & $6,8 \mathrm{a}$ & 2,7 & $-12,0 a$ & 5,7 \\
\hline Rosinha $\mathrm{G}_{2}$ & $1,4 \mathrm{a}$ & 0,7 & $-7,5 \mathrm{a}$ & 0,4 & $3,0 \mathrm{bc}$ & 1,3 & $-8,6 a$ & 1,7 \\
\hline Carioca & $1,7 \mathbf{a}$ & 0,4 & $-5,4 b$ & 0,3 & $5,5 \mathrm{ab}$ & 1,8 & $-7,7 \mathrm{a}$ & 3,8 \\
\hline Aroana & $1,3 b$ & 0,4 & $-4,7 \mathrm{c}$ & 0,5 & $2,4 \mathrm{c}$ & 1,4 & $-9,2 a$ & 3,8 \\
\hline Moruna & $1,4 \mathrm{a}$ & 0,4 & $-3,4 d$ & 0,6 & $2,9 c$ & 1,9 & $-11,2 \mathrm{a}$ & 2,5 \\
\hline$r$ & $0,13 \mathrm{~ns}$ & & & & $0,30 \mathrm{~ns}$ & & & \\
\hline
\end{tabular}

s: desvio-padrão; r: coeficiente de correlação entre acúmulo de prolina e $\psi \mathrm{p}$.

Letras não comuns in dicam diferenças significativas pelo teste de Tukey a 5\%.

No quadro 3 são apresentados os dados de acúmulo de prolina livre e potencial de pressão de ágùa de folhas de cultivares de feijão submetidos a temperatura de 5 a $10^{\circ} \mathrm{C}$ durante dois e onze dias: o acúmulo de prolina foi praticamente semelhante em todos os cultivares testados após dois ou onze dias de escassez de água; o mesmo pode ser dito em relação ao potencial de água da folha. Quando se comparam os dados do quadro 1 com os do 3, observa-se um comportamento inverso em relação à medida do $\psi_{\mathrm{p}}$, uma vez que, no primeiro, houve uma elevação acentuada no teor de prolina $\mathrm{e} \psi_{\mathrm{p}}$ quando o periodo de escassez de água passou de dois para oito dias, o que 
não foi observado no segundo caso, indicando que, a baixas temperaturas, pode ter ocorrido a diminuição nos processos metabólicos sem a diminuição na água disponível.

QUADRO 2 - Comprimento médio e respectivo desvio-padrão (s) de plantas de feijoeiro submetidas a 8 dias de estresse hídrico, medido aos 26 (6 dias de estresse), 33 (5 dias após reidratação) e 39 dias de idade das plantas

\begin{tabular}{|c|c|c|c|c|c|c|c|c|c|c|}
\hline \multirow{3}{*}{$\begin{array}{l}\begin{array}{c}\text { Idade } \\
\text { das } \\
\text { plantas }\end{array} \\
\text { dias }\end{array}$} & \multicolumn{10}{|c|}{ Cultivares } \\
\hline & \multicolumn{2}{|c|}{ Jalo } & \multicolumn{2}{|c|}{ Rosinha $G_{2}$} & \multicolumn{2}{|c|}{ Carioca } & \multicolumn{2}{|c|}{ Aroana } & \multicolumn{2}{|c|}{ Moruna } \\
\hline & $\mathrm{cm}$ & s & $\mathrm{cm}$ & $\mathrm{s}$ & $\mathrm{cm}$ & $\mathbf{s}$ & $\mathrm{cm}$ & $\mathbf{s}$ & $\mathrm{cm}$ & s \\
\hline 26 & 16,1 & 3,3 & 17,0 & 5,9 & 16,7 & 3,1 & 16,6 & 3,8 & 11,8 & 2,2 \\
\hline 33 & 18,6 & 3,1 & 19,5 & 7,2 & 19,2 & 3,9 & 19,0 & 5,5 & 13,2 & 2,7 \\
\hline 39 & 37,7 & 8,1 & 28,1 & 5,2 & 31,9 & 8,9 & 28,2 & 8,9 & 19,8 & 5,4 \\
\hline
\end{tabular}

QUADRO 3 - Acúmulo de prolina livre e potencial de pressão de água $\left(\psi_{\mathrm{p}}\right)$ de folhas de cultivares de feijoeiro submetidas a temperaturas de 5 a $10^{\circ} \mathrm{C}$ durante. .11 dias

\begin{tabular}{lccccc}
\hline \multirow{2}{*}{ Cultivar } & \multicolumn{3}{c}{ Dias de tratamento de $5 \mathrm{a} 10^{\circ} \mathrm{C}$} \\
\cline { 2 - 3 } \cline { 5 - 6 } & \multicolumn{2}{c}{2} & & \multicolumn{2}{c}{11} \\
\cline { 2 - 3 } \cline { 5 - 6 } & Prolina & $\psi_{\mathrm{p}}$ & & Prolina & $\psi_{\mathrm{p}}$ \\
\hline \multirow{2}{*}{ Jalo } & $\mu \mathrm{mol} / \mathrm{g}$ & $\mathrm{atm}$ & $\mu \mathrm{mol} / \mathrm{g}$ & $\mathrm{atm}$ \\
Rosinha $\mathrm{G}_{2}$ & 0,02 & $-2,8$ & & 0,04 & $-1,8$ \\
Carioca & 0,01 & $-2,5$ & & 0,02 & $-1,5$ \\
Aroana & 0,01 & $-3,1$ & & 0,04 & $-1,7$ \\
Moruna & 0,01 & $-3,0$ & & 0,01 & $-1,5$ \\
& 0,01 & $-3,2$ & 0,02 & $-1,5$ \\
\hline
\end{tabular}

Submetendo-se cultivares de feijão a temperaturas de 2 a $5^{\circ} \mathrm{C}$, durante onze dias no estádio de crescimento - entre 23 e 34 dias após plantio (DAP), e treze dias no florescimento - 67 a 80 DAP, quando se determinou o teor de prolina, foram utilizados os dados cujos valores médios para teor deste aminoácido são apresentados no quadro 4. Não se verificou diferença significativa para cultivares no primeiro período estudado, 23 a 34 DAP, porém isso ocorreu para o período de treze dias a partir do flo- 
rescimento, ao se considerar teor de prolina livre. A altura total foi semelhante para todos os cultivares, variando de 39 a $42 \mathrm{~cm}$, in dicando não ter o crescimento da planta influenciado o acúmulo de aminoácido. Essas temperaturas, 2 a $5^{\circ} \mathrm{C}$, indicaram que deve ter diminuído o movimento da água do solo para as raízes da planta, com a conseqüen te diminuição do potencial de pressão de água nas folhas. $O$ potencial de água nas folhas $\left(\psi_{p}\right)$ apresentou uma diminuição em relação ao acréscimo do acúmulo de prolina, o que é contrário ao obtido por $\mathrm{CHU}$ et alii $(1974,1978)$ para cevada a $5^{\circ} \mathrm{C}$, indicando que esta é mais adaptada às condições frias que o feijoeiro.

QUADRO 4 - Acúmulo de prolina livre ( $\mu \mathrm{mol} / \mathrm{g}$ de matéria seca) em folhas de feijoeiro submetidas a temperaturas de $2 \mathrm{a}^{\circ} \mathrm{C}$ por 11 dias durante o período de crescimento (23 a 34 DAP) e 13 dias quando do florescimento (67 a 80 DAP)

\begin{tabular}{lcc}
\hline Cultivar & $\begin{array}{c}\text { Período 23 a 34 dias } \\
\text { após plantio }\end{array}$ & $\begin{array}{c}\text { Período 67 a 80 dias } \\
\text { após plantio }\end{array}$ \\
\cline { 2 - 3 } & Prolina & Prolina \\
Rosinha $\mathrm{G}_{2}$ & $\mu \mathrm{mol} / \mathrm{g}$ & $\mu \mathrm{mol} / \mathrm{g}$ \\
Bico de Ouro & $4,9 \mathrm{a}$ & $4,6 \mathrm{a}$ \\
Roseli & $4,4 \mathrm{a}$ & $4,1 \mathrm{ab}$ \\
Jalo & $3,7 \mathrm{a}$ & $3,3 \mathrm{ab}$ \\
Moruna & $3,4 \mathrm{a}$ & $3,3 \mathrm{ab}$ \\
Curitibano & $4,1 \mathrm{a}$ & $3,0 \mathrm{ab}$ \\
P-511 & $3,5 \mathrm{a}$ & $2,3 \mathrm{~b}$ \\
\hline média atm & $3,5 \mathrm{a}$ & $2,3 \mathrm{~b}$ \\
\hline
\end{tabular}

Letras năo comuns indicam diferenças significativas pelo teste de Tukey a 5\%.

Comparando os dados dos quadros 1 e 4, verifica-se que os cultivares Jalo e Moruna tiveram comportamento semelhante em temperaturas bai$x$ as, condição essa desfavorável ao crescimento avan tajado do 'Jalo'. $O$ estresse a baixa temperatura, por ter efeito imediato sobre o crescimento, parece mais interessante para indicar a possível resistência de plantas à escassez de água, visto o resultado apresentado pelo 'P-511', considerado resistente à seca e baixa temperatura.

Segundo LEVITT (1972), no resfriamento de 1 a $5^{\circ} \mathrm{C}$ ocorre a solidificação dos lipídios, levando à formação de 'buracos' na membrana lipoprotéica celular, que perde a permeabilidade. Posteriormente, ocorre a perda 
de solutos pelo plasmalema, desidratação da célula, desagregação das proteínas pela formação de grupos dissulfatos, sendo os processos fisiológicos alterados. Nas membranas mitocondriais, há diminuição da cadeia respiratória, falta de ATP e inibição da síntese protéica, com acúmulo de compostos amoniacais e aminoácidos livres. Em temperaturas altas e desidratação, ocorre a hidrólise e degradação protéica, com formação de compostos amoniacais e aminoácidos livres e maior evaporação, levando ao fechamento dos estômatos e diminuição da fotossíntese. Portanto, plantas tolerantes ao frio, também o são à seca ou ao calor, pois o efeito de temperaturas extremas e déficits de água são difíceis de separar.

No quarto experimento, que constou da medida do teor de prolina e do potencial de água das folhas após catorze dias de estresse hídrico a temperatura ambiente, verificou-se comportamento diferencial entre cultivares. 'Rosinha $\mathrm{G}_{2}$ ' apresentou maior valor absoluto de aumento de prolina livre que os demais; entretanto, estatisticamente, foi semelhante ao 'Roseli'. Este áltimo, mais 'Bico-de-Ouro', 'Jalo' e 'Moruna' apresentaram valores intermediários, e 'Curitibano' e 'P-511', os menores acúmulos, comprovando que este aminoácido está associado à disponibilidade interna de água nas plantas, pois - 'P-511' e 'Curitibano' apresentaram os menores acúmulos de prolina e os maiores $\psi_{\mathrm{p}}$, indicand o maior manutençao de água, conferindo-lhes a resistência à seca e baixa temperatura. A correlação entre prolina e $\psi_{\mathrm{p}}$ foi significativa e indicou que quanto maior o acúmulo de prolina, menor o $\psi_{\mathrm{p}}$ (Quadro 5).

QUADRO 5 - Acúmulo de prolina livre em folhas de cultivares de feijoeiro de plantas ño irrigadas por 14 dias em temperatura ambiente

\begin{tabular}{lcc}
\hline Cultivar & Prolina & $\psi_{\mathrm{p}}$ \\
\hline & $\mu \mathrm{mol} / \mathrm{g}$ & $\mathrm{atm}$ \\
Rosinha $\mathrm{G}_{2}$ & $15,78 \mathrm{a}$ & $-19,3$ \\
Roseli & $10,69 \mathrm{a}$ & $-13,5$ \\
Bico de Ouro & $10,53 \mathrm{~b}$ & $-11,7$ \\
Jalo & $8,78 \mathrm{bc}$ & $-11,6$ \\
Moruna & $7,04 \mathrm{bc}$ & $-8,0$ \\
Curitibano & $5,82 \mathrm{c}$ & $-7,3$ \\
P-511 & $4,52 \mathrm{c}$ & $-5,0$ \\
$\mathrm{r}$ & $0,99 * *$ & \\
\hline
\end{tabular}

Letras não comuns indicam diferenças significativas pelo teste de Tukey a 5\%. r: coeficiente ou correlação entre acúmulo de prolina e potencial de pressão de água $\left(\psi_{\mathrm{p}}\right)$. 


\title{
4. CONCLUSOES
}

1. O acúmulo de prolina está correlacionado com o potencial de água na planta.

2. Em temperaturas de 5 a $10^{\circ} \mathrm{C}$, houve diminuição da quantidade de prolina e elevação do potencial de pressão de água nas folhas da planta (em relação ao experimento utilizando temperatura ambiente).

3. Em temperaturas de 2 a $5^{\circ} \mathrm{C}$, verificou-se acréscimo da prolina acumulada e diminuição do potencial de pressão de água na planta, independente de seu estádio de desenvolvimento quando submetida a déficit hídrico.

4. Os cultivares Curitibano e P-511 apresentaram menor acúmulo de prolina e maior potencial de pressão de água na planta, confirmando a hipótese de que é possível o reconhecimento de material genético resistente à escassez de água através da medida do acúmulo de prolina.

\author{
SUMMARY \\ TEMPERATURE, RELATIVE HUMIDITY AND WATER AVAILABILITY ON \\ PROLINE ACCUMULATION OF DRY BEANS
}

Four experiments were performed in growth chamber and greenhouse at Campinas, in 1982, to study the relations of dry bean cultivars submmited to period of water and temperature stress. Adaptation of plants to stress conditions was measured through proline accumulation and water potential $(\psi \mathrm{p})$ in leaves. In all tested cultivars proline content showed a straight negative correlation with water potential in leaves. Cultivars P-511 and Curitibano showed lower proline content and higher water potential than the other under study, this confirming the hypothesis that it is possible to identify materials genetically resistant to water stress by selection of plants or lines with low proline content.

Index terms: temperature, water and humidity; proline; water potential.

\section{REFERENCIAS BIBLIOGRÁFICAS}

BOGGESS, S.F.; STEWART, C.R. \& KOEPPE, D.E. Oxidation of proline by plant mitochondria. Plant Physiology, 62:22-25, 1978.

CHU, T.M.; ASPINALL, D. \& PALEG, L.G. Stress metabolism. VI. Temperatures stress and the accumulation of proline in barley and radish. Australian Journal of Plant Physiology, 1:87-97, 1974.

;JUSAITIR, M.; ASPINALL, D. \& PALEG, G. Accumulation of free proline at low temperatures. Physiologia Plantarum, 43:254-260, 1978. 
HANSON, A.D.; NELSEN, C.E. \& EVERSON, E.H. Evaluation of free proline accumulation as an index of drought resistance using two contrasting barley cultivars. Crop Science, 17(5):720-726, 1977.

; PEDERSEN, A.R. \& EVERSON, E.H. Capacity for proline accumulation during water stress in barley and its implications for breeding for drough resistance. Crop Science, 14:489-493, 1979 a.

\& TULLEY, E.R. Proline accumulation in water-stressed barley leaves in relation to translocation and the nitrogen budget. Plant Physiology, 63(3):518-523, 1979b.

HUANG, A.HC. \& CAVALIERI, A. I Proline oxidase and water-stress induced proline accumulation in Spinach leaves. Plant Physiology, 63:531$535,1979$.

JAGER, H.I. \& MEYER, H.R. Effect of water stress on growth and proline metabolism of Phaseolus vulgaris L. Oecologia, Berlin, 30:83-96, 1977.

LEVITT, I. Responses of plants to environmental stress. In: THE MEASUREMENT of drought resistance. New York, Academic Press, 1972. p.425-445.

RENA, A.B. \& MASCIOTTI, G.Z. The effect of dehydration on nitrogen metabolism and growth of 4 bean cultivars (Phaseolus vulgaris L.). Revista Ceres, Viçosa, 23(128):288-301, 1976.

SAWAZAKI, H.E.; TEIXEIRA, J.P.F. \& ALMEIDA, L.D.A. Estresse de água no crescimento, produ tividade e acúmulo de prolina em feijão. Bragantia, Campinas, 40:157-166, $1981 \mathrm{a}$.

Variação do teor de prolina em folhas de feijão em função da disponibilidade de água no solo. Bragantia, Campinas, 40:47-56, $1981 \mathrm{~b}$.

SINGH, T.N.; ASPINALDI, D.; PALEG, L.G. \& BOGGESS, S.F. II. Changes in proline metabolism and concentration in excised plant tissues. Australian Journal Biological Science, 26:57-63, 1973.

STEWART, C.R. Role of carbohydrates in proline accumulation in wilted barley leaves. Plant Physiology, 61:775-778, 1978.

; BOGGESS, S.F.; ASPINALDI, D. \& PALEG, L.G. Inhibition of proline oxidation by water stress Plant Physiology, 59:930-932, 1977. 